\title{
The Things That Unite Us
}

\author{
Michael James Heron ${ }^{1}$ (D)
}

Received: 18 January 2018/Accepted: 6 March 2018/Published online: 16 April 2018

(C) Springer Science+Business Media, LLC, part of Springer Nature 2018

We are living through something of a golden age in video game scholarship. A discipline which was once maligned and misrepresented has burgeoned into a rich and vibrant field of inquiry that attracts some of the most interesting scholarship to be found in academia. Games are economically important, but they are also of considerable cultural value (Heron 2016). We have now shown, as a body of practise, that games are also intellectually stimulating. There are journals on game criticism, on game culture, on game development and on many other intersections of games and academic fields. Those that felt there was no academic merit to the topic have long been discredited. Those who saw the potential in video game studies from the start have been vindicated. We can all bask in the warmth of a job well done. We collectively constructed a citadel of study and defended it from those that would seek to tear it down.

That is a considerable accomplishment, and one about which we should feel proud. However, it seems, when reflecting upon the discipline as it stands, that in our efforts to defend it we may have inadvertently excluded many topics that would have benefited from being situated within the walls we have built. In our haste to cement the relevance of video game studies as an academic discipline, we have concentrated more on the video part than we have on the game.

Many of the game journals that exist put barriers in place for those looking to publish scholarly work on other forms of gaming. Sometimes it's in the name of the outlet, such as with The Computer Games Journal you are reading now. Sometimes it's in the scope or the aims. Sometimes it's in the expertise of the editorial panel, and most often it's in the context of the work that is published. Such things are an inevitable outcome of academic specialism, but they have had the effect of depriving us of a richer and more interesting environment in what is sometimes

Michael James Heron

m.j.heron1@rgu.ac.uk

1 Robert Gordon University, Aberdeen AB10 7QB, Scotland, UK 
known as ludology. We conduct close readings of video games; discuss the mechanisms which they can be constructed and deconstructed; explore the optimal techniques that can be employed in their development; and, consider the economic context in which they function. Even relatively obscure genres can be discussed safely, provided they can be situated in a digital context. Currently, there are few credible options for publishing academically impactful scholarly work on analog games.

Increasingly, I have come to think of this as a missed opportunity. My own recent studies over the past 2 years have been focused on tabletop games, and specifically the accessibility of tabletop games. This has been the single most interesting project of my academic life to date-it's been like stumbling on a rich vein of gold to find that absolutely nobody is mining. However, it's been hard to square this personal enthusiasm with my professional incentives-I have visited a rich and foreign land, but there is nowhere to sell the silks I have gathered there. As such, the primary output of this project to date has been a research blog (http://meeplelikeus.co.uk), which generates no citations, accrues little research kudos, and ultimately serves as a repository of data-points rather than a substantive academic output. I have found myself often cast in the role of ranting prophet. I enthuse, trying to convince others of the value of work which has been explicitly, even if only unconsciously, marginalized by the nascent evolution of the discipline of game studies. These are games, they are interesting games, and they have a lot to reveal to us if we investigate. Before I fell in love with tabletop gaming as a focus of academic inquiry, I believed I couldn't be surprised by games any more. I felt that my years of experience as an academic and enthusiast had given me sufficient grounding in the topic that I was incapable of feeling shock or delight. Imagine how it feels when you've become numb to novelty to open up a game box and simply marvel at what you find. Analog games offer interactions that can encompass the whole of human experience and sensory input. There are games focused entirely on conversations, games that use touch and smell as core mechanisms of play, and games that use the ingenuity of humans as inventive components in game state generation. I have found not just a domain for gaming that is phenomenally rich in its manifestations, but also a domain for accessibility that continues to delight me. I am excited by the work I do in this field because not only is it underexplored-in many cases it is completely unexplored, certainly in an academic context.

When I took on the editorial duties for this special issue of the Computer Games Journal, I made a specific request that the scope of the journal be widened to permit games of all types to be covered. That not only provided me a chance to show off the rich spices I have gathered on my travels. It is primarily because my own studies have convinced me that there are stories and tales of equal value to be found if we broaden our exploration to include manifestations of gaming we have previously excluded. Imagine a paper on the accessibility issues of Live Action Roleplaying, or on the psychologies of players during an RPG. Imagine how the design lessons of modern tabletop games might inform the prototyping phases of digital games, or how the streamlined on-boarding processes of modern video games might enhance the often cumbersome task of learning a new board game rule set. Imagine how we might explore the prosocial impact of communication games, or how the social 
context of physical gaming might serve to alleviate issues of loneliness or exclusion. Video games have increasingly refined their focus on the lone individual, where social interaction is mediated through distance. As that has happened, tabletop games have doubled down on the face-to-face nature of play. Tabletop games are interesting because people are interesting, and we have in many ways lost the focus on people as video games have evolved. Even as multiplayer games have become more popular, community has degraded. We no longer sit playing split-screen with our families - we manage the landscape of our social lives through layers of digital abstraction. We've gained in quantity, but sacrificed quality. We've gained ease of participation, but lost all the intimacy.

I believe that the future of videogame studies focuses less on the video part and more on the game. I hope that the relaxed focus of this special issue on game accessibility is a sign of things to come-that we can see as a discipline the potential for being more accommodating in the scope of the games we discuss. We have lessons that video games could impart to tabletop games. Lessons that LARPs could impart to video games. Things from RPGs that could inform board games, and things from board games that could enhance video games. There is a bright, exciting conversation to be had in academia about games in all their multifaceted manifestations-we just need to be willing to have the discussions. That in turn needs us to be permissive-more than anything else, we need to encourage scholars within this discipline that these are discussions they should think about starting.

\section{Reference}

Heron, M. J. (2016). Cultural integration and the accessibility of gaming. The Computer Games Journal, 5, 91-94. https://doi.org/10.1007/s40869-016-0028-x. 